\title{
Level of kisspeptin-10 in patients with multiple sclerosis and the association between third ventricle diameter size and vitamin $D$ level
}

\author{
T. AKDAG ${ }^{1 \dagger *}$ (D) A.U. UCA ${ }^{2 \dagger}$, M. $\operatorname{ALTAS}^{2 \dagger}$, F.O. $\operatorname{ODABAS}^{3 \dagger}$ and F. AKTAS ${ }^{4 \dagger}$
}

\author{
${ }^{1}$ Meram Vocational School, Necmettin Erbakan University, Konya, Turkey \\ ${ }^{2}$ Department of Neurology, Faculty of Medicine, Necmettin Erbakan University, Konya, Turkey \\ ${ }^{3}$ Department of Neurology, Konya Education and Research Hospital, University of Health Science, \\ Konya, Turkey \\ ${ }^{4}$ Department of Neurology, Abdulkadir Yuksel Hospital, Gaziantep, Turkey
}

Received: November 1, 2020 - Accepted: January 19, 2021

Published online: June 16, 2021

(๔ 2021 Akadémiai Kiadó, Budapest

\begin{abstract}
Objective: Multiple sclerosis (MS) is a chronic and progressive neurological disease affecting the central nervous system (CNS). Some studies report an association between MS pathogenesis and cytokines. Here, we aimed to determine and evaluate serum kisspeptin-10 level in MS patients and its related clinic parameters. Materials and Methods: A total of 92 participants, 46 patients with relapsing-remitting MS (mean age, 38.92 $\pm 14.76 ; 22$ men and 24 women) and 46 healthy controls (mean age, $37.04 \pm 15.49 ; 22$ men and 24 women) were enrolled in the study. All MS patients were neurologically examined, and magnetic resonance imaging (MRI) was performed. Clinical data (neuropathic pain, expanded disability status scale (EDSS) score, etc.) and the patients' demographic characteristics were recorded. The serum level of kisspeptin-10 was analyzed by enzyme-linked immunosorbent assay (ELISA) method. Results: The level of kisspeptin-10 was measured as $2.305 \pm 2.781 \mathrm{ng} / \mathrm{mL}$ in MS patients and $9.342 \pm 9.483 \mathrm{ng} / \mathrm{mL}$ in controls. MS patients had significantly lower kisspeptin-10 levels than controls $(P=0.000)$. Kisspeptin-10 has the highest diagnostic value [Area under curve $(\mathrm{AUC})=0.881,95 \%$ confidence interval $(\mathrm{CI}), 0.812-0.950$ ] as cut-off value (2.470), sensitivity $(80.40 \%)$ and specificity $(72.87 \%)$ in the MS group. Furthermore, the kisspeptin-10 level was negatively correlated with third ventricle diameter (TVD) $(P=0.048)$ and vitamin $\mathrm{D}$ concentration $(P=0.004)$. No significant difference was determined between kisspeptin-10 and other
\end{abstract}

\footnotetext{
${ }^{*}$ Corresponding author. Meram Vocational School, Necmettin Erbakan University, Konya, Turkey. Tel.: +90 505 65973 50. E-mail: turanakdag570@gmail.com
}

${ }^{\dagger}$ All authors contributed equally to the study. 
clinical parameters. Conclusion: As a potential prognostic biomarker, serum kisspeptin-10 level was significantly lower in patients with MS than in those without. Moreover, we observed negative correlations between vitamin D, TVD size, and kisspeptin-10. We think comprehensive studies are needed to verify and elucidate this issue.

\section{KEYWORDS}

kisspeptin-10, multiple sclerosis, neurological disease, third ventricle, vitamin D

\section{INTRODUCTION}

As a progressive neurological disease, multiple sclerosis (MS) is defined by perivenular inflammatory lesions and demyelinating plaques within the central nervous system (CNS) [1]. Typical symptoms of MS are observed in patients between 20 and 40 years of age (about 60$65 \%)$. MS symptoms are usually diagnosed between 15 and 50 years of age, and the prevalence of the disease in women is about twice that in men [2,3].

Free radicals, neurogenic inflammation, and oxidative stress have important roles in the pathogenesis of MS [4]. Although its underlying causes still remain unclear, the disease is often characterized by environmental factors such as Epstein-Barr virus infection, vitamin D deficiency, exposure to ultraviolet B light, smoking, and obesity [5]. Based on the literature, MS is considered to be an autoimmune disease, partially related to genetic susceptibility and environmental factors [6]. Evidence has shown that the progression of MS is correlated with the abnormally low or high expression of certain cytokines. A previous study indicated that the levels of tumor necrosis factor-alpha (TNF $\alpha$ ) were increased in the cerebrospinal fluid (CSF) of MS patients and related to the progression and severity of the disease [7]. In the study by Vranova et al. [8], however, it was determined that CSF and serum levels of cystatin C were significantly lower in MS patients than in the controls. Currently, various protocols, including McDonald criteria, CSF analyses, magnetic resonance imaging (MRI), etc. are used to diagnose MS in clinical procedures. However, there are no effective biomarkers for the diagnosis, prognosis, and evaluation of MS. The identification of sensitive and predictive biomarkers may provide advantages in terms of early diagnosis and lower costs for clinical trials.

Kisspeptin, which is a neuropeptide of the arginine-phenylalanine (RF) amide family, is encoded by the kiss- 1 gene and involved in many physiological events such as the initiation of puberty, the regulation of prolactin release, glucose homeostasis, and anti-metastatic and antioxidative effects $[9,10]$. The gene of kisspeptin (kiss-1) is located at 1q32.1 in humans and consists of three introns and four exons [11]. The first product of this gene is kisspeptin-145 (145 amino acid protein/Kp145). After the proteolytic cleavage of the precursor protein, Kp145 generates a 54-amino acid-amidated peptide (Kp54), and then shorter degradation fragments are formed, such as Kp14, Kp13, and Kp10 (Kisspeptin-10) [12]. All these fragments of kisspeptin are capable of binding to the coupled-G protein receptor (GPR54/Kiss1R) and play significant roles in the regulation of the hypothalamic-pituitary-gonad axis, as well as the inhibition of proliferation, cell motility, chemotaxis, metastasis and invasion [13]. 
Messenger RNAs (mRNA) of kisspeptins and kiss1R are expressed especially in the brain, hypothalamus, spinal cord, liver, placenta, cardiovascular system and lymph nodes [14]. During stress and inflammation, there is a decrease in kisspeptin and kiss1R expression, and also insufficiency in kisspeptin responsiveness [15]. Kisspeptin-10, the most bioactive fragment, is well-known as a potent anticoagulant, antitumor, angiogenic and vasoconstrictive neuropeptide [16]. However, the data about the function and role of kisspeptin-10 in neurological diseases are very limited.

Based on the literature, there is little or no information regarding the relationship between MS and kisspeptin-10. This study was performed to examine whether the serum level of kisspeptin-10 is associated with MS, and there also is a relationship between kisspeptin-10 and disease-related clinic parameters.

\section{MATERIALS AND METHODS}

A total of 92 participants admitted to the Neurology Department between July and October 2020 were enrolled in the study. The study was composed of 46 MS patients (mean age: $38.92 \pm 14.76$, 22 men and 24 women) diagnosed with relapsing-remitting MS (RRMS) in the attack-free period through magnetic resonance imaging (MRI). MRI studies have shown focal lesions with contrast enhancement in the arterial phase and decreased contrast in the late venous phase. The healthy control group was composed of 46 participants (mean age: 37.04 $\pm 15.49,22$ men and 24 women) without any neurological disease or history of MS. Female participants in both the patient and healthy control groups were in the pre-menopausal period (mean age: $37.00 \pm 9.43$ and $37.42 \pm 9.13$, respectively).

The exclusion criteria included subjects with the following: chronic heart failure, renal failure, thyroid disease, mental illnesses, endocrinopathies, cardiovascular diseases, diabetes mellitus, pregnancy, neurologic disease, and those with a history of any gynecological disorders. The expanded disability status scale (EDSS) was performed in MS patients, and the rating intensity for scores ranged from 0 (no disability) to 10 (maximum disability) [17]. The sizes of the third ventricle diameter (TVD) and pituitary diameter (PD) of MS patients were measured by an experienced radiologist using a 1.5-Tesla MRI scanner (Siemens, Germany). The brain MRI protocol including axial T1-weighted turbo spin-echo (linear resolution: $0.1 \mathrm{~mm}$, gap: $1.5 \mathrm{~mm}$, and slice thickness: $5 \mathrm{~mm}$ ) was used to measure TVD. The three-dimensional (3D) spectroscopic imaging sequence was used to acquire the localized data focused on the hypothalamus region through point resolved spectroscopy (PRESS) $(\mathrm{TE} / \mathrm{TR}=30 / 1,500 \mathrm{~ms}, \mathrm{FOV}$ was $10 \times 10$ $\times 8$, interpolated to $16 \times 16 \times 8 \mathrm{~cm}^{3}$ with a nominal voxel size of $10 \times 10 \times 12.5 \mathrm{~mm}^{3}$ and volumes of interest (VOIs) $50 \times 60 \times 35 \mathrm{~mm}^{3}, 7 \mathrm{~min}$ acquisition and elliptical weighting). In each patient, TVD was defined as the maximum transverse dimension of TVD between the tuberal regions of the hypothalamus. T1-weighed MP-RAGE dataset was reoriented to the AC$\mathrm{PC}(\mathrm{AC}=$ anterior commissure, $\mathrm{PC}=$ posterior commissure $)$ plane. The trans-axial slice of 2 $\mathrm{mm}$ below the anterior commissure was selected. After denoising, up-sampling, and the segmentation of MR images, TVD was defined as the maximum transverse dimension of TVD between the tuberal regions of the hypothalamus [18]. The dimensions of PD were taken as the maximum distance in $\mathrm{mm}$ between lateral and superoinferior surfaces, whereas PD was obtained by multiplying the height $\times$ depth $\times$ width $\times 0.52$ [19]. The estimations of TVD and 
PD were expressed as $\mathrm{mm}$ and $\mathrm{mm}^{3}$, respectively. Body mass index (BMI) was also calculated as weight $(\mathrm{kg})$ divided by the square of the height $(\mathrm{m})$. Fatigue was measured using the fatigue severity scale (FSS) as a range scale from 1 (strongly disagree) to 7 (strongly agree) [20]. Additionally, the questionnaire of Douleur Neuropathique 4 questions (DN4) was performed to evaluate neuropathic pain in MS patients [21]. MS patients had received no vitamin D supplements and medications for at least 6 months before the investigation. Blood samples were collected in anticoagulant-free tubes from each participant, and such features as age, sex, medical history, and other clinical information were recorded. Also, the samples of both female groups were taken in the non-menstrual period. After immediate centrifugation at $2.000 \times \mathrm{rpm}$ (at $4{ }^{\circ} \mathrm{C}$ for $20 \mathrm{~min}$ ), serum aliquots of $1 \mathrm{~mL}$ were frozen immediately at $-80^{\circ} \mathrm{C}$ and kept until the assay. The present study was approved by the ethics committee of Necmettin Erbakan University Medicine Faculty, Turkey, and conformed to the ethical guidelines of the Declaration of Helsinki in 1961 and later amendments. Written informed consent was obtained from all participants before the enrollment.

\section{Measurements of serum kisspeptin-10 and vitamin D levels}

Serum concentrations of kisspeptin-10 were assessed by an immunoenzymatic method with the commercially available enzyme-linked immunosorbent assay (ELISA) kits according to the manufacturer's instructions (Human Kisspeptin-10 Elisa Kit, standard curve range: $0.05 \mathrm{ng} / \mathrm{mL}$ $10 \mathrm{ng} / \mathrm{mL}$, sensitivity: $0.02 \mathrm{ng} / \mathrm{mL}$, intra-assay CV: $8 \%$; interassay CV: $10 \%$; catalog no. E3919Hu, Bioassay Technology Laboratory, Shanghai, China). The absorbance of the samples was measured by a microtiter plate reader at $450 \mathrm{~nm}$ (ELx800TM, Bio-Tech Instruments, USA), and the levels of kisspeptin-10 were expressed in units of ng $/ \mathrm{mL}$. Serum levels of vitamin D were assessed by chemiluminescence immunoassay (CLIA) method with the commercially available Architect $25-\mathrm{OH}$ vitamin D kit and measured by Abbott Architect i1000SR immunoassay analyzer. The levels of vitamin D were also expressed in units of $\mathrm{ng} / \mathrm{mL}$.

\section{Statistical analysis}

Statistical analysis was performed with the Statistical Package for the Social Sciences (SPSS) software package, version 22.0 (SPSS Inc, Chicago, IL, USA). The statistical significance of the difference between the variables was tested using the Mann-Whitney U-test. Correlations were analyzed with the Spearman rank correlation coefficient test. Receiver operating characteristic (ROC) curves were generated by plotting the sensitivity against 1-specificity, and the cut-off values were determined by Youden's index method. The data were expressed as mean \pm standard deviation (SD). Statistical significance was defined as $P<0.05$.

\section{RESULTS}

All the characteristics of MS patients and healthy controls are shown in Table 1. In the study, the mean ages of 46 patients with MS and 46 healthy controls were found as 38.92 and 37.04 years, respectively. The serum kisspeptin-10 level was significantly lower in the patients with MS than in those without $(2.305 \pm 2.781 \mathrm{ng} / \mathrm{mL}$ and $9.342 \pm 9.483 \mathrm{ng} / \mathrm{mL}$, respectively; $P=0.000)$. No significant correlation was found between kisspeptin-10 levels and EDSS score, disease duration, 
Table 1. Comparison of socio-demographics, serum kisspeptin-10 level and other clinical findings between MS patients and healthy controls

\begin{tabular}{|c|c|c|c|}
\hline Parameters & MS group $(n=46)$ & Healthy group $(n=46)$ & $\mathrm{P}$ \\
\hline Age $(y)$, mean & $38.92 \pm 14.76$ & $37.04 \pm 15.49$ & 0.129 \\
\hline Male & $40.35 \pm 7.38$ & $37.75 \pm 8.66$ & 0.442 \\
\hline Female & $37.00 \pm 9.43$ & $37.42 \pm 9.13$ & 0.826 \\
\hline \multicolumn{4}{|l|}{ Sex } \\
\hline Male (n) & $22(47.82 \%)$ & $22(47.82 \%)$ & \multirow[t]{2}{*}{-} \\
\hline Female (n) & $24(52.18 \%)$ & $24(52.18 \%)$ & \\
\hline \multicolumn{4}{|l|}{ Kisspeptin-10 (ng/mL) } \\
\hline Mean/Median & $2.305 \pm 2.781 / 1.795$ & $9.342 \pm 9.483 / 3.845$ & $0.000^{*}$ \\
\hline Male & $2.52 \pm 3.97$ & $6.42 \pm 7.09$ & $0.000^{*}$ \\
\hline Female & $2.18 \pm 1.84$ & $11.59 \pm 10.56$ & $0.000^{*}$ \\
\hline BMI $\left(\mathrm{kg} / \mathrm{m}^{2}\right)$ & $25.513 \pm 4.081$ & $23.437 \pm 2.36$ & 0.167 \\
\hline EDSS score $(0-10)$ & $1.945 \pm 1.841$ & N/A & - \\
\hline Disease duration (y) & $8.869 \pm 6.259$ & N/A & - \\
\hline \multirow[t]{4}{*}{ Number of attacks } & (1-3) $20 \%, 45.3$ & \multirow[t]{4}{*}{ N/A } & \multirow[t]{4}{*}{-} \\
\hline & (4-7) $16 \%, 36.3$ & & \\
\hline & $(8-10) 4 \%, 9.2$ & & \\
\hline & $(>10) 4 \%, 9.2$ & & \\
\hline Neuropathic pain & $4.043 \pm 3.210$ & N/A & - \\
\hline Fatigue $(1-7)$ & $5.826 \pm 2.885$ & N/A & - \\
\hline TVD (mm) & $5.163 \pm 1.909$ & N/A & - \\
\hline $\mathrm{PD}\left(\mathrm{mm}^{3}\right)$ & $257.244 \pm 18.631$ & N/A & \\
\hline Vitamin D (ng/mL) & $26.154 \pm 18.631$ & N/A & - \\
\hline
\end{tabular}

BMI: Body mass index, EDSS: Expanded disability status scale, MS: Multiple sclerosis, NA: Not applicable, PD: Pituitary diameter, TVD: Third ventricle diameter. Data were presented as mean \pm standard deviation, and descriptive statistics were calculated via the Mann-Whitney $U$ test. *A P value $<0.05$ was considered to be statistically significant.

Table 2. Correlations of serum kisspeptin-10 levels with other clinical findings in MS patients including correlation analyses and Spearman's rank correlation

\begin{tabular}{lrr}
\hline & \multicolumn{2}{c}{ Kisspeptin-10 } \\
\cline { 2 - 3 } Parameters & \multicolumn{1}{c}{$r$} & $P$ \\
\hline Disease duration & -0.164 & 0.276 \\
Number of attacks & 0.099 & 0.512 \\
Neuropathic pain & -0.106 & 0.485 \\
Fatigue & -0.058 & 0.753 \\
TVD (mm) & -0.293 & $0.048^{*}$ \\
PD $\left(\mathrm{mm}^{3}\right)$ & 0.070 & 0.644 \\
EDSS score & -0.157 & 0.297 \\
Vitamin D & -0.182 & $0.004^{*}$ \\
\hline
\end{tabular}

EDSS: Expanded disability status scale, MS: Multiple sclerosis, TVD: Third ventricle diameter, PD: Pituitary diameter. ${ }^{*}$ A P value $<0.05$ was considered to be statistically significant. 
Table 3. Correlations of kisspeptin-10 serum levels of MS patients concerning other clinical findings in terms of female and male

\begin{tabular}{lrrrr}
\hline & \multicolumn{2}{c}{ Kisspeptin-10 } \\
\cline { 2 - 5 } Parameters & \multicolumn{2}{c}{ Female } & $P$ & Male \\
\cline { 2 - 5 } & \multicolumn{1}{c}{$r$} & 0.987 & $r$ \\
\hline Disease duration & 0.00 & 0.449 & -0.44 & 0.078 \\
Number of attacks & 0.15 & 0.338 & 0.10 & 0.692 \\
Neuropathic pain & -0.18 & 0.992 & 0.01 \\
Fatigue & 0.00 & $0.031^{*}$ & -0.15 & 0.555 \\
TVD (mm) & -0.40 & 0.343 & -0.10 & 0.708 \\
PD (mm ${ }^{3}$ ) & 0.18 & 0.697 & -0.35 & 0.165 \\
EDSS score & 0.08 & $0.002^{*}$ & -0.65 & 0.007 \\
Vitamin D & -0.55 & -0.37 & 0.142 \\
\hline
\end{tabular}

EDSS: Expanded disability status scale, MS: Multiple sclerosis, TVD: Third ventricle diameter, PD: Pituitary diameter. *A $P$ value $<0.05$ was considered to be statistically significant.
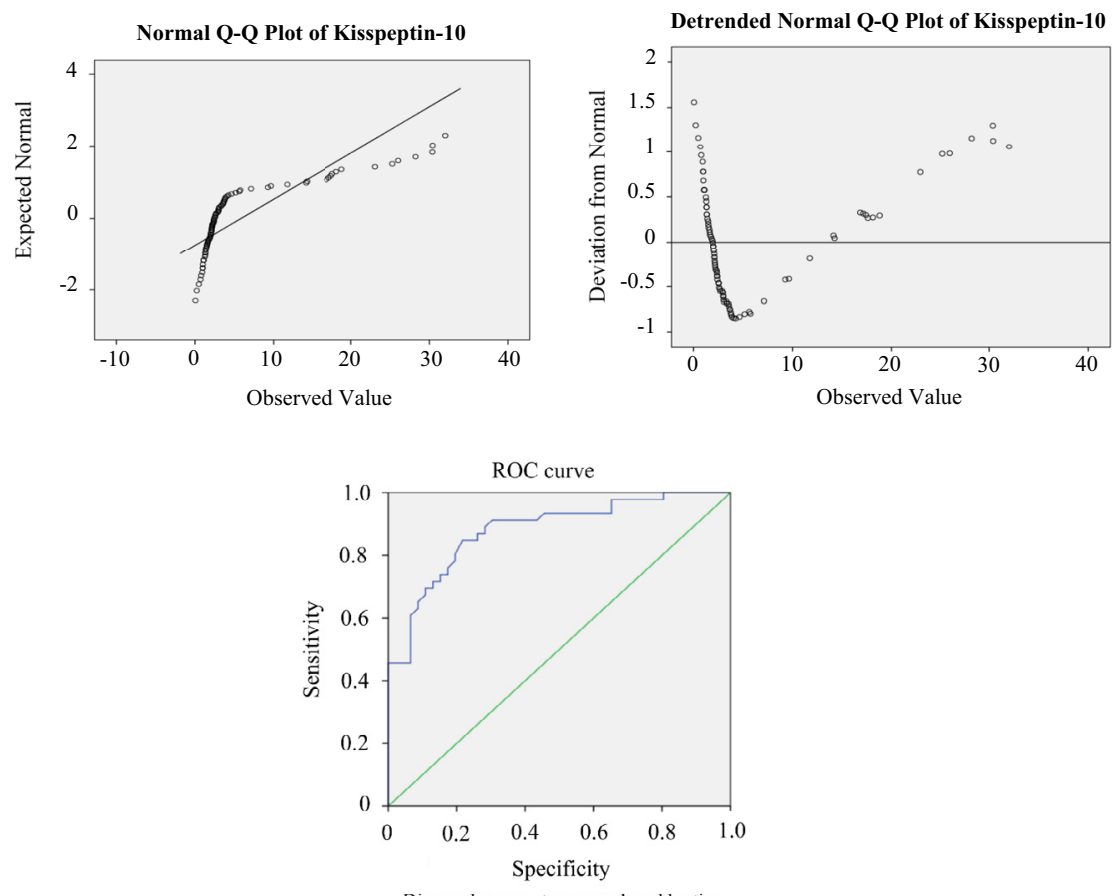

Diagonal segments are produced by ties

\begin{tabular}{|c|c|c|c|c|c|c|}
\hline Factor & $\begin{array}{c}\text { AUC } \\
(95 \% \mathrm{CI})\end{array}$ & $\begin{array}{c}\text { Cut-off } \\
\text { value }\end{array}$ & $\begin{array}{c}\text { Sensitivity } \\
(\%)\end{array}$ & $\begin{array}{c}\text { Specificity } \\
(\%)\end{array}$ & PPV & NPV \\
\hline Kisspeptin-10 & $\begin{array}{c}0.881 \\
(0.812-0.950)\end{array}$ & 2.470 & $80.40 \%$ & $72.87 \%$ & $80.69 \%$ & $74.31 \%$ \\
\hline
\end{tabular}

AUC: Area under the curve, CI: Confidence interval, MS: Multiple sclerosis, NPV: Negative predictive value, PPV: Positive predictive value

Fig. 1. ROC curve and predictive power of the serum kisspeptin-10 concentration in MS patients and normal-detrended plots of serum kisspeptin-10 
number of attacks, neuropathic pain, fatigue, and PD. As demonstrated in Table 2, a negative correlation was found between kisspeptin-10 levels and [22] TVD size $(r=-0.293, P=0.048)$ and [15] vitamin $\mathrm{D}(r=-0.182, P=0.004)$. As shown in Table 3, a negative correlation was found between kisspeptin-10 levels in female MS patients and [22] TVD size $(r=-0.40, P=$ $0.031)$ and [15] vitamin $\mathrm{D}(r=-0.55, P=0.002)$. ROC curve analysis and plots of kisspeptin10 are shown in Fig. 1.

\section{DISCUSSION}

The pathophysiology and etiology of MS, an incompletely known, complex disease remain elusive. As an inflammatory and demyelinating disease, the condition may lead to severe physical and cognitive impairments due to various neurological defects in CNS [23]. The immunopathogenesis of MS involves a process triggered by $\mathrm{CD} 4^{+} \mathrm{T} 4$ cells against CNS antigens and supported by B cells, resulting in demyelination of oligodendrocyte and loss of axons [24]. Although the pathogenesis of axonal loss and demyelination is not fully understood, the effects of inflammatory cytokines, such as nitric oxide, interleukin-1 (IL-1) beta, TNF-alpha and matrix metalloproteinases, and other effects produced by $\mathrm{CD} 4^{+}$and $\mathrm{CD}^{+} \mathrm{T}$ cells, active microglia/ macrophages, and complement proteins play important roles in these processes [25].

Novel proteins and cytokines have been investigated for the diagnosis, prognosis and clinical evaluation in MS. The redundancy or deficiency in the levels of these biomarkers may provide valuable contributions to many diseases. Therefore recent studies have focused on revealing the relationship between newly identified proteins and possible related diseases. In a recent study, it has been observed that serum tau (a phosphoprotein) and decreased brain-derived neurotrophic factor (BDNF) levels increased to a higher extent among MS patients than controls [26]. It has also been proposed that serum levels of tau and BDNF may be used as biomarkers of the severity of MS. In a study by Alpua et al. [22] high serum levels of apelin-13 were reported in MS patients, compared with the controls. Another study also revealed that serum cystatin $\mathrm{C}$ levels of MS patients were significantly lower than those in the controls $(P=0.008)$ [27]. In our study, serum kisspeptin-10 levels were significantly decreased in the MS group, compared to the controls $(2.305 \pm 2.781$ and $9.342 \pm 9.483 \mathrm{ng} / \mathrm{mL}$, respectively, $P=0.000)$.

In a study, TVD diameters in MS patients and controls were determined as $3.9 \pm 1.6 \mathrm{~mm}$ and $3.4 \pm 0.8 \mathrm{~mm}$, respectively [28]. Additionally, because of determining the increased size of TVD in MS patients $(6.2 \pm 1.7 \mathrm{~mm})$, d'Alessandro et al [29]. proposed that the increase in third ventricle diameter may represent a criterion of positivity of neurological disease in those with chronic cerebrospinal venous insufficiency. Moreover, a recent study has reported that TVD measurements below the hypothalamic sulcus may point to hypothalamic atrophy [18]. In our study, TVD measurements were performed to determine the extent of hypothalamic atrophy, and PD was also measured due to the neuroendocrine connection between the hypothalamus and pituitary. As shown in Table 1, the sizes of TVD and PD were measured in MS patients below the hypothalamic sulcus as $5.163 \pm 1.909 \mathrm{~mm}$ and $257.244 \pm 18.63 \mathrm{~mm}^{3}$, respectively. There was also a significant correlation between TVD size and kisspeptin-10 $(r=-0.293, P=$ 0.048). Even so, no significant correlation was found between PD size and kisspeptin-10 level ( $P$ $=0.644$ ) (Table 2). In female MS patients, a negative correlation was found between kisspeptin10 levels and TVD size $(r=-0.40, P=0.031)$ (Table 3$)$. 
The overall immunomodulatory effects of vitamin $\mathrm{D}$ on different categories of $\mathrm{B}$ and $\mathrm{T}$ lymphocytes and several cytokines have been well-established in recent years. In many immunological studies conducted in MS patients, it was demonstrated that vitamin D is likely to affect MS via various immunomodulatory mechanisms. However, vitamin D leads to many beneficial immunological effects such as the reduction of IL-10 cytokines, pro-inflammatory Th17 lymphocytes, the weakening of the harmful cytokine IL17, and B cell immunoreactivity. Several studies have shown that vitamin D levels are lower in MS patients than those in the controls. Hatamian et al [30]. reported that serum levels of vitamin D were determined to be lower in MS patients, compared to healthy controls $(26.5 \pm 16.3 \mathrm{ng} / \mathrm{mL}$ and $37.1 \pm 19.7 \mathrm{ng} /$ $\mathrm{mL}$, respectively, $P=0.003$ ). In the present study, serum vitamin $\mathrm{D}$ levels were found as $26.154 \pm 18.631 \mathrm{ng} / \mathrm{mL}$ in MS patients. Also, there was a negative correlation between vitamin $\mathrm{D}$ and kisspeptin-10 $(r=-0.182, P=0.004)$ (Table 2). As shown in Table 3, a negative correlation was found between kisspeptin-10 level and vitamin D in female MS patients $(r=$ $-0.55, P=0.002)$.

A wide variety of MS-induced chronic symptoms may cause spasms, spasticity, fatigue, bladder dysfunction and pain [31]. The most common type is usually burning, stinging, pain, or central neuropathic pain defined as paroxysmal neuralgia. In clinical practice, the most commonly used scales for the assessment of MS are visual/numerical analog scales and EDSS, based on the subjective evaluation of the patients [32]. In our study, there was no significant association between kisspeptin-10 level and EDSS score, neuropathic pain and fatigue in MS patients (Table 2).

To the best of our knowledge, no data are available on circulating levels of kisspeptin-10 in those with MS so far, and our study is the first to investigate the kisspeptin-10 level in MS patients. Further research in the field of potential biomarkers for MS should be directed towards an earlier and more accurate diagnosis, as well as targeted and individualized therapeutic approach with minimal intervention and economic cost. As a result, kisspeptin-10 may be a valuable prognostic tool in the MS disease. We consider that further comprehensive studies with a larger sample size should be conducted to verify our findings.

Ethics committee approval: This study was approved by the Ethics Board of the Meram Faculty of Medicine of Necmettin Erbakan University in Konya, Turkey (Approval number: 2020/2597$68)$.

Financial support: None.

Conflict of interest: The authors report no conflicts of interest.

\section{ACKNOWLEDGMENTS}

We would like to thank all of the study participants, as well as the staff of the Neurology Department of Meram Medical Faculty of Necmettin Erbakan University. The authors also thank Numan Duran for assistance with language editing. 


\section{REFERENCES}

1. Popescu BF, Pirko I, Lucchinetti CF. Pathology of multiple sclerosis: where do we stand. Continuum (MinneapMinn). 2013; 19(4): 901-21.

2. Gilroy J. Basic neurology. 3rd edition. New York-St.Louis-San Francisco: McGraw-Hill; 2000. pp. 199-223.

3. Rolak LA. Multiple sclerosis: it's not the disease you thought it was. Clin Med Res 2003; 1(1): 57-60. https:// doi.org/10.3121/cmr.1.1.57.

4. Solleiro-Villavicencio H, Rivas-Arancibia S. Effect of chronic oxidative stress on neuroinflammatory response mediated by $\mathrm{CD}^{+}{ }^{+}$Cells in neurodegenerative diseases. Front Cell. Neurosci. 2018; 12:114. https://doi.org/ 10.3389/fncel.2018.00114.

5. Ascherio A. Environmental factors in multiple sclerosis. Expert Rev Neurother 2013; 13(12 Suppl.): 3-9. https://doi.org/10.1586/14737175.2013.865866.

6. Waubant E, Lucas R, Mowry E, Graves J, Olsson T, Alfredsson L, et al. Environmental and genetic risk factors for MS: an integrated review. Ann Clin Transl Neurol 2019; 6(9): 1905-22. https://doi.org/10.1002/acn3. 50862.

7. Sharief MK, Hentges R. Association between tumor necrosis factor-alpha and disease progression in patients with multiple sclerosis. N Engl J Med 1991; 325(7): 467-72.

8. Vranová HP, Sládková V, Mareš J, Hluštík P, Langová J, Kanovský P. Cystatin C as a marker of degeneration in multiple sclerosis. Neurology 2013; 80(7): P03.241.

9. Olaniyan OT, Meraiyebu AB, Auta KB, Dare JB, Anjorin YD, Shafe MO. Kisspeptin system: a multi-homeostatic system. IOSR J Pharm Biol Sci 2013; 5(4): 87-101.

10. Poling MC, Quennell JH, Anderson GM, Kauffman AS. Kisspeptin neurons do not directly signal to RFRP-3 neurons but RFRP-3 may directly modulate a subset of hypothalamic kisspeptin cells in mice. J Neuroendocrinol 2013; 25(10): 876-86.

11. Zeydabadi Nejad S, Ramezani Tehrani F, Zadeh-Vakili A. The role of kisspeptin in female reproduction. Int J Endocrinol Metab 2017; 15(3): e44337.

12. Ohtaki T, Shintani Y, Honda S, Matsumoto H, Hori A, Kanehashi K, et al. Metastasis suppressor gene KiSS-1 encodes peptide ligand of a G-protein-coupled receptor. Nature 2001; 411(6837): 613-7.

13. Olbrich T, Ziegler E, Türk G, Schubert A, Emons G, Gründker C. Kisspeptin-10 inhibits bone-directed migration of GPR54-positive breast cancer cells: evidence for a dose-window effect. Gynecol Oncol 2010; 119(3): 571-8.

14. Kirby HR, Maguire JJ, Colledge WH, Davenport AP. International union of basic and clinical pharmacology. LXXVII. kisspeptin receptor nomenclature, distribution, and function. Pharmacol Rev 2010; 62(4): 565-78.

15. Attard CC. The role of kisspeptin signaling in the hypothalamic-pituitary-gonadal axis. Malta Med J 2017; 29(3): 30-6.

16. Sato K, Shirai R, Hontani M, Shinooka R, Hasegawa A, Kichise T, et al. Potent vasoconstrictor kisspeptin-10 induces atherosclerotic plaque progression and instability: reversal by its receptor GPR54 antagonist. J Am Heart Assoc 2017; 6(4): e005790. https://doi.org/10.1161/JAHA.117.005790.

17. Kurtzke JF. Rating neurologic impairment in multiple sclerosis: an expanded disability status scale (EDSS). Neurology 1983; 33(11): 1444-52.

18. Polacek H, Kantorova E, Hnilicova P, Grendar M, Zelenak K, Kurca E. Increased glutamate and deep brain atrophy can predict the severity of multiple sclerosis. Biomed Pap Med Fac Univ Palacky Olomouc Czech Repub 2019; 163(1): 45-53. 
19. Roldan-Valadez E, Garcia-Ulloa AC, Gonzalez-Gutierrez O, Martinez-Lopez M. 3D volumetry comparison using $3 \mathrm{~T}$ magnetic resonance imaging between normal and adenoma-containing pituitary glands. Neurol India $2011 ; 59(5): 696-9$.

20. Krupp LB, LaRocca NG, Muir-Nash J, Steinberg AD. The fatigue severity scale: application to patients with multiple sclerosis and systemic lupus erythematosus. Arch Neurol 1989; 46(10): 1121-3.

21. Krause SJ, Backonja MM. Development of a neuropathic pain questionnaire. Clin J Pain 2003; 19(5): 306-14.

22. Alpua M, Turkel Y, Dag E, Kisa U. Apelin-13: a promising biomarker for multiple sclerosis. Ann Indian Acad Neurol 2018; 21(2): 126-9.

23. Ghasemi N, Razavi S, Nikzad E. Multiple Sclerosis: pathogenesis, symptoms, diagnoses, and cell-based therapy. Cell J 2017; 19(1): 1-10. https://doi.org/10.22074/cellj.2016.4867.

24. Racke MK. Immunopathogenesis of multiple sclerosis. Ann Indian Acad Neurol 2009; 12(4): $215-20$.

25. Gold R, Wolinsky JS. Pathophysiology of multiple sclerosis and the place of teriflunomide. Acta Neurol Scand 2011; 124(2): 75-84.

26. Islas-Hernandez A, Aguilar-Talamantes HS, Bertado-Cortes B, Mejia-delCastillo GJ, Carrera-Pineda R, Cuevas-Garcia CF, et al. BDNF and Tau as biomarkers of severity in multiple sclerosis. Biomark Med 2018; 12(7): 717-26. https://doi.org/10.2217/bmm-2017-0374.

27. Isgrò MA, Caldarella C, Scribano D, Colacicco L, Giannace A, Zuppi C, et al. Is there a role for serum cystatin $\mathrm{C}$ as a biomarker of multiple sclerosis. Biochim Clin 2014; 38(3): 218-22.

28. Muller M, Esser R, Kötter K, Voss J, Müller A, Stellmes P. Third ventricular enlargement in early stages of multiple sclerosis is a predictor of motor and neuropsychological deficits: a cross-sectional study. BMJ Open 2013; 3:e003582.

29. d'Alessandro A, Mandolesi S, Niglio T, Orsini A, Di Cello P, Pelle F, et al. Increased size of third ventricle in patients with multiple sclerosis and chronic cerebrospinal venous insufficiency. J Vasc Diagn 2014; 2:91-7.

30. Hatamian H, Bidabadi E, Seyed Saadat SM, Saadat NS, Kazemnezhad E, Ramezani H, et al. Is serum vitamin D levels associated with disability in patients with newly diagnosed multiple sclerosis. Iran J Neurol 2013; 12(2): 41-6.

31. Kesselring J, Beer S. Symptomatic therapy and neurorehabilitation in multiple sclerosis. Lancet Neurol 2005; 4(10): 643-52. https://doi.org/10.1016/S1474-4422(05)70193-9.

32. Meyer-Moock S, Feng YS, Maeurer M, Dippel FW, Kohlmann T. Systematic literature review and validity evaluation of the expanded disability status scale (EDSS) and the multiple sclerosis functional composite (MSFC) in patients with multiple sclerosis. BMC Neurol 2014; 14:58. https://doi.org/10.1186/1471-2377-1458. 